\title{
NEAR FIELD OPTICAL MICROSCOPY AND SPECTROSCOPY WITH STM AND AFM PROBES
}

\author{
O. Bergossi*, R. Bachelot, H. Wioland, G. Wurtz, R. Laddada \\ P.M. ADAM, J.L. BiJeon AND P. ROYer \\ Laboratoire de Nanotechnologie et d'Instrumentation Optique \\ Universitè de Technologie de Troyes \\ 12 rue Marie Curie, BP 2060, 10010 Troyes Cedex, France
}

\begin{abstract}
This article deals with a new generation of scanning near field optical microscopes (SNOM), called apertureless SNOM, based on metallic, semi-conductive or dielectric probes. The classification of the apertureless probe among the usual SNOM probes is discussed in the first part. Then, we present the different apertureless SNOM configurations that we develop, with various commercial AFM and home-made tungsten tips, and several illumination and collection modes. Finally, after a preliminary result in near field imaging, we propose a promising application of such microscopes dedicated to the near field fluorescence spectroscopy.
\end{abstract}

PACS numbers: 07.60.Pb, 42.62.Fi, 61.16.Ch, 68.35.Bs

\section{Introduction}

In the family of the scanning probe microscopy (SPM), the scanning near field optical microscope (SNOM) offers some original characteristics. Particularly, the use of photon as the information vector makes the SNOM a unique tool for a large variety of applications. These applications are generally an improvement of classical optical techniques in sample characterization and manufacture (microscopy, spectroscopy, or lithography for instance [1]). The improvement concerns the lateral resolution, which is limited in far field at half the wavelength $\lambda$ by the diffraction of light. This resolution has been brought in the nanometric range $(\lambda / 10-\lambda / 30)$ by taking advantage of the local interaction between three elements: the studied sample, an incident light source and a material probe (size $\ll \lambda$ ) located at a small distance $(\ll \lambda)$ from the sample surface. The far field detection of the intensity of the propagating waves generated by this complex interaction provides the SNOM signal.

*e-mail: olivier.bergossi@univ-troyes.fr 
In Sec. 2, we propose a classification of the usual SNOM probes. Especially, the ability of commercial or home-made SPM probes for SNOM devices is discussed. In the next section, we briefly present the various experimental configurations that we test (in reflection or transmission), with a significant result in microscopy. In the last part, we introduce some experiments scheduled in near field optical spectroscopy.

\section{Probes for scanning near field optical microscopy}

\subsection{Classification of the probes}

Historically, the first SNOM probe applied the idea of a nano-aperture: a hole on a perfectly plane opaque screen, generating evanescent waves [2]. The evolution of the technique gave rise to a way now classical to manufacture the SNOM probe, based on a tapered optical fiber. The tapered zone of the fiber is metal coated, except at the very end, thus creating a nanometric hole. This hole either allows a local illumination of the sample surface (illumination mode), or picks-up and collects the near field above the surface (collection mode). This system is thus called "aperture SNOM".

Besides the role of the tip end, the optical fiber guides the light from the laser source towards the tip (illumination mode), or guides the collected light towards a remote photodetector (collection mode).

The most convivial scanning mode is the constant distance mode (in particular to avoid the tip damaging on the sample surface), by help of a feedback signal linked to the surface topography. Unfortunately, except in total internal reflection configurations [3], the near field optical signal is improper as feedback signal (it is not directly linked to the topography). Consequently, several combinations of SNOM + SPM were developed, and new configurations are regularly mentioned. These combinations are based either on the above-mentioned specific SNOM probes, or on usual or modified SPM probes, if able to provide a complementary near field optical sensitivity. The first solution (specific SNOM probes) is generally associated with a shear force microscope [1]. The second solution (SPM existing or modified probes) gives rise to a new category of probes, called the perturbative (or apertureless [4]) probe. In its principle, the tip locally perturbs the optical near field which is diffracted in various directions. An apertureless probe acts as a Mie-Rayleigh particle, diffracting the evanescent waves lying on the sample surface. Let us note that the apertureless probe does not necessarily guide the diffracted light. A classical optical system (objective or fiber) is generally in charge of the diffracted light collection. Consequently, the apertureless probe can be made of semi-conductive or metallic (as well as dielectric) material.

\subsection{Apertureless probes}

In our laboratory, we develop apertureless SNOM devices using either various commercial dielectric or semi-conductive AFM probes, or home-made metallic (tungsten) probes. Advantages of perturbative probes, compared to specific SNOM probes, are of several kinds. Firstly, the sharper probe extremity allows a lateral resolution about 10 times better than in the case of specific optical probes [4]. 
Secondly, as no guiding property by the probe is necessary, the evolution towards infrared light up to $10.6 \mu \mathrm{m}$ is allowed, especially for tungsten probes whose end (assimilated to a sphere) has a polarizability almost constant from ultraviolet to middle-infrared wavelengths [5]. Thirdly, the commercial SPM tips are more reproducible (in terms of shape and extremity size) than the specific optical probes. This point is of great importance for SNOM characterization and image interpretation [6].

Our home-made tungsten tip (obtained by chemical etching) is similar to classical STM tungsten (W) probes, but with some specificities. In particular, the shape angle is very low, to minimize the shadow effect on the illumination beam [7]. Moreover, the tip is curved, and used as an AFM probe (Fig. 1). Figure 1 indicates some characteristics of our W probe (general shape - (a), resonance frequency - (b), and proves its ability for topography imaging in AFM mode - (c). The typical stiffness constant and resonance frequency are in the $20-150 \mathrm{~N} / \mathrm{m}$ and $4-6 \mathrm{kHz}$ ranges, respectively. The final radius size is about $15 \mathrm{~nm}$.

(a)

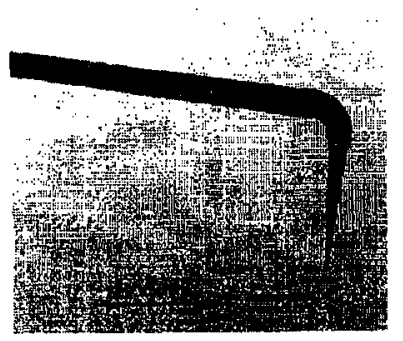

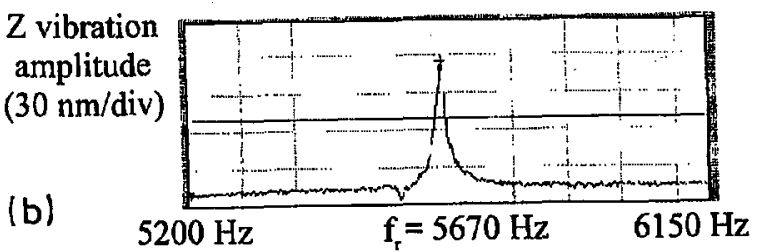

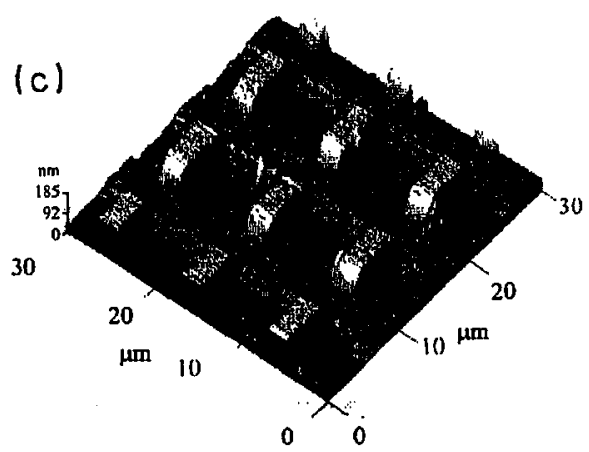

Fig. 1. Home-made tungsten (W) probes: successful use, as AFM probes, by the commercial SPM. (a) $2 \mathrm{~mm} \times 1.5 \mathrm{~mm}$ snapshot of a $\mathrm{W}$ probe (cantilever and tip), (b) measurement of the resonance frequency, (c) $30 \mu \mathrm{m} \times 30 \mu \mathrm{m}$ intermittent contact mode AFM image of a test grating.

Furthermore, the ability of the home-made tungsten probe for a STM + SNOM device is in progress, in collaboration with the Institute of Physics, Polish Academy of Sciences, in Warsaw. 


\section{Imaging at a sub-wavelength resolution with an apertureless SNOM}

We have implemented three apertureless SNOM devices, developed from the CP, "stand-alone", and M5 SPM models from Park Scientific Instruments (PSI) ${ }^{\dagger}$. The three set-ups allow nine configurations (five in reflection mode and four in transmission mode), see Fig. 2.

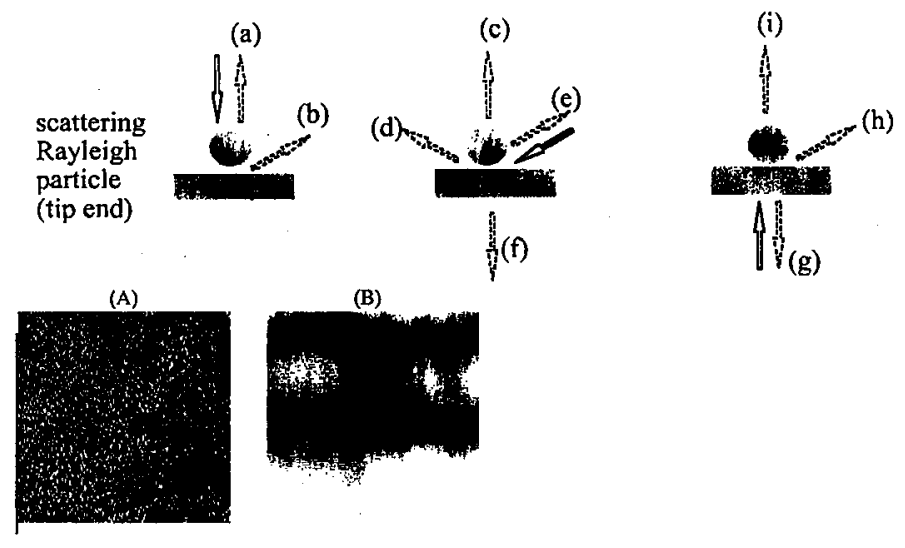

Fig. 2. Apertureless SNOM configurations allowed by the three set-ups of our laboratory. Reflection mode and transmission mode are possible. The solid-line and dashed-line arrows represent the direction of the incident light and the detected light, respectively. $(\mathrm{a}-\mathrm{b})$ : normal incidence. (a) normal detection, (b) oblique detection. (c-f): oblique incidence. (c) normal detection, (d) oblique detection, (e) back-scattering configuration, (f) normal detection beneath the sample. (g-i): normal incidence beneath the surface sample. (g) normal detection beneath the sample, (h) oblique detection above the sample surface, (i) normal detection above the sample surface. (A-B): example of images obtained by the configuration (a). AFM (A) and SNOM (B) images (size $2 \mu \mathrm{m} \times 2 \mu \mathrm{m}$ ) of the front facet of a laser diode, obtained with a silicon-nitride (SiN) probe, at $\lambda=635 \mathrm{~nm}$. A pure dielectric contrast is revealed.

Each set-up enables the control of the polarization state of both incident and detected light, and uses various types of laser sources and probes: various shapes (pyramidal, conical, and tetrahedral) and materials (silicon-nitride: SiN, silicon: $\mathrm{Si}$, tungsten: W). For each configuration, the tip vibrates (at a frequency $f$ near the resonance frequency) perpendicularly to the surface sample, and the SNOM signal is provided by a lock-in detection at the frequency $f$. This procedure allows one to extract, from the total intensity received by the final photo-detector, the small part which corresponds to the light scattered by the local interaction between the tip

${ }^{\dagger}$ A description of these set-ups can be found in several papers which we have submitted for publication. For instance, the set-up developed from the M5 model of PSI allows five SNOM configurations and is described in: G. Wurtz, R. Bachelot, P. Royer, submitted to Rev. Sci. Instrum. The set-up developed from the CP model is presented in: P.M. Adam, P. Royer, R. Laddada, J.L. Bijeon, submitted to Appl. Opt. 
end, the sample and the incident light. In the case of a SNOM+AFM combination, the "non-contact" (or "attractive") and the "tapping" (or "intermittent contact") AFM modes are used.

This polyvalence enables a number of experimental studies to perform, including polarization contrasts, influence of the illumination and detection angles, interpretation of the SNOM signal formation, influence of the tip material and shape on the SNOM image. Recent theoretical works (e.g. [8]) have demonstrated that the study of these parameters and physical effects is of great importance for the characterization of the apertureless SNOM devices.

Figures $2 \mathrm{~A}$ and $\mathrm{B}$ present a couple of images, obtained by the configuration (a) of Fig. 2, with a silicon-nitride (SiN) probe. They are the simultaneous AFM (A) (in intermittent contact mode) and SNOM (B) images ( $2 \mu \mathrm{m} \times 2 \mu \mathrm{m}$ ) of the front facet of a laser diode. The sample does not present any topographic contrast (the crystal facet is cleaved), but optical index variations (various GaAs, $\mathrm{GaAl}_{x} \mathrm{As}$ layers). Figure $2 \mathrm{~A}$ indicates a very flat surface (roughness $=2 \mathrm{~nm}$ RMS) whereas Fig. 2B reveals a complementary dielectric contrast, corresponding to various layers, with a spatial resolution much better than $\lambda / 2$. Figure $2 \mathrm{~B}$ is a significant example of contrast detectable by near field optics but not by the AFM.

\section{Spectroscopy with apertureless SNOM}

In addition to its microscopic function, the SNOM can operate in the spectroscopic mode, to perform a local chemical analysis of surfaces. Especially, fluorescence spectroscopy is a widespread technique used in many fields like biology and chemistry. The first near field fluorescence images of single molecules have been obtained by Betzig et al. [9] by using an aperture SNOM. More recently, Martin et al. [4] have reported preliminary results in scattering spectroscopy with an apertureless SNOM. In our laboratory, we are preparing fluorescence measurements with the apertureless SNOM configurations and probes described above. The test
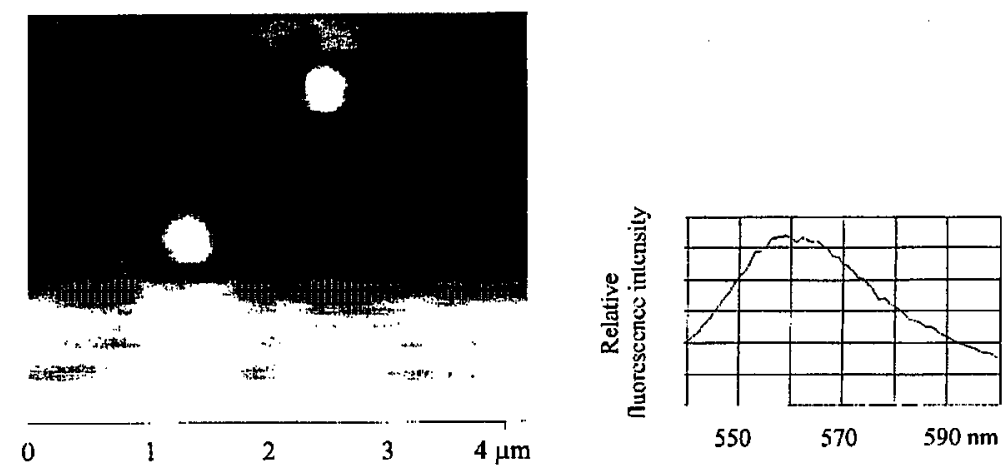

Fig. 3. AFM image of two separated rhodamine doped polystyrene spheres deposited on a glass substrate. The apparent diameter is $300 \mathrm{~nm}$. Up left: fluorescence emission spectrum in far field, with a laser excitation at $\lambda=530 \mathrm{~nm}$ (horizontal axis in nanometers). 
sample is formed by small rhodamine-doped polystyrene spheres deposited on a glass substrate, and excited at $\lambda=530 \mathrm{~nm}$. An AFM image of this sample is presented in Fig. 3, showing two separated polystyrene spheres. The fluorescence emission spectrum in far field (Fig. 3 up left) gives spatially averaged information on large sample zones (about $100 \mu \mathrm{m}$ ), while the near field spectrum should provide more localized information, down to the single molecule detection.

\section{Conclusion}

The preliminary results validate our apertureless SNOM devices in near field optical microscopy of opaque as well as transparent samples, with a sub-wavelength lateral resolution. The SNOM images provide information complementary to a simultaneous AFM topography. More generally, the literature,reveals that tungsten STM tips [5, 7], and also AFM commercial tips of various shapes and materials [4] (see the footnote in Sec. 3) have proved their ability as probes for apertureless SNOM. These results lead us to perform optical spectroscopy experiments with the same probes soon, at a similar spatial resolution, using our various apertureless SNOM configurations.

\section{References}

[1] M.A. Paesler, P.J. Moyer, Near Field Optics, John Wiley \&Sons Inc., New York 1996.

[2] D.W. Pohl, W. Denk, M. Lanz, Appl. Phys. Lett. 44, 651 (1984).

[3] D. Courjon, K. Sarayeddine, M. Spajer, Opt. Commun. 71, 23 (1989).

[4] Y. Martin, F. Zenhausern, H.K. Wickramasinghe, Appl. Phys. Lett. 68, 2475 (1996).

[5] A. Lahrech, R. Bachelot, P. Gleyzes, A.C. Boccara, Opt. Lett. 21, 1 (1996).

[6] D. Barchiesi, D. Van Labeke, Microsc. Microanal. Microstruct. 5, 1 (1994).

[7] R. Bachelot, P. Gleyzes, A.C. Boccara, Appl. Opt. 36, 2160 (1997).

[8] A. Madrazo, R. Carminati, M. Nieto-Vesperinas, J.J. Greffet, J. Opt. Soc. Am. A 15, 109 (1998).

[9] E. Betzig, R.J. Chichester, Science 262, 1422 (1993). 